absolutely uniform. The motion produced by this modification of the Klepsydra is communicated by a cord to a pulley on the projecting end of the axis of a vertical cylinder enclosed in a brass box at the end of the observing telescope. A strip of photographic celluloid film is wrapped around the circumference of the cylinder, and is thus moved just behind and very close to a narrow slit near the focus. It is believed that satisfactory results will be obtained in this way, as the friction and mass of the moving parts are reduced to a minimum.

The.difficulty of obtaining a perfectly smooth motion is very great, as my experience with various forms of clocks and sliding plate-holders taugth me last year, and I consider it the fatal objection to the methods of Dr. Braun and Dr. Lohse as published. They are both excellent in theory, but if put in practice they would probably be met with mechanical difficulties of a serious nature. Dr. von Konkoly states that Dr. Lohse's apparatus has been in use at Potsdam, but the reproduction he gives of a photograph of the chromosphere is rendered of little value by just such inequalities of motion as the form of apparatus makes it practically impossible to avoid. (Himmelsphotographie pag. 346.)

In conclusion, I wish to express my appreciation of many suggestions given by Dr. Braun in his paper in Poggen. dorff's Annalen, and though some of these had already occurred to me others will undoubtedly prove useful in my work. The $>$ Berichte von dem Haynald Observatorium in Kalocsar is not at my disposal, and I have had to depend upon the description of the modified apparatus given by Dr. von Konkoly in the first edition of his Himmelsphotographie.. My second method is certainly very similar in principle to Dr. Braun's, but the means employed have no points in common, and each possesses certain advantages, Dr. Braun's, I think, being rather the better of the two. But this method was with me afterthought, and it does not compare in practicability with my first method as described above.

Kenwood Physical Observatory, Chicago, 189 I Febr. 26.

George E. Hale.

\title{
Osservazioni lunari
}

al Circolo Meridiano dell' Osservatorio astronomico di 'Torino.

Lo strumento adoperato è quello di Reichenbach acquistato da Plana sul principio del 1820 ed è identico a quello descritto da Bessel nella sesta sezione della raccolta delle sue osservazioni. L'oculare ha ingrandimento 129. Il cratere Mösting A, descritto dal Sig. J. Franz, è facilmente riconoscibile in un bel cerchietto e se ne possono osservare al cronografo gli appulsi dei due bordi allo stesso filo. Ma nelle osservazioni ad orecchio quali sono queste che presento ho sempre registrato l'appulso del centro del cratere.

I tempi dell' osservazione sono segnati dal pendolo normale Dent, al quale si riferiscono i $\Delta t$. Il micrometro é composto di 9 fili verticali $e$ si ha avuto cura di ottenere, nelle sere in cui si possono osservare lembo e cratere, completo ai 9 fili il passaggio del cratere. Il circolo durante queste osservazioni si è sempre lasciato ad ovest.

Dal 17 Novembre 1890 ad oggi il tempo a Torino si è mantenuto molto nebbioso e coperto per cui nelle due lunazioni si sono potute fare poche osservazioni.

Ad ogni osservazione corrisponde una determinazione di tempo e degli errori strumentali con stelle del Berliner Jahrbuch contemporanea all' osservazione stessa. Le ascensioni rette delle stelle di culminazione lunare e della Luna furono ricavate dal Nautical Almanac, quelle del cratere dalle effemeride date dal Sig. J. Franz nell' A. N. 2997.

Dedotte le ascensioni rette del centro della Luna dalle stelle di culminazione lunare trovo le differenze Osservazione meno Nautical Almanac che sono registrate nell' ultimo quadro.

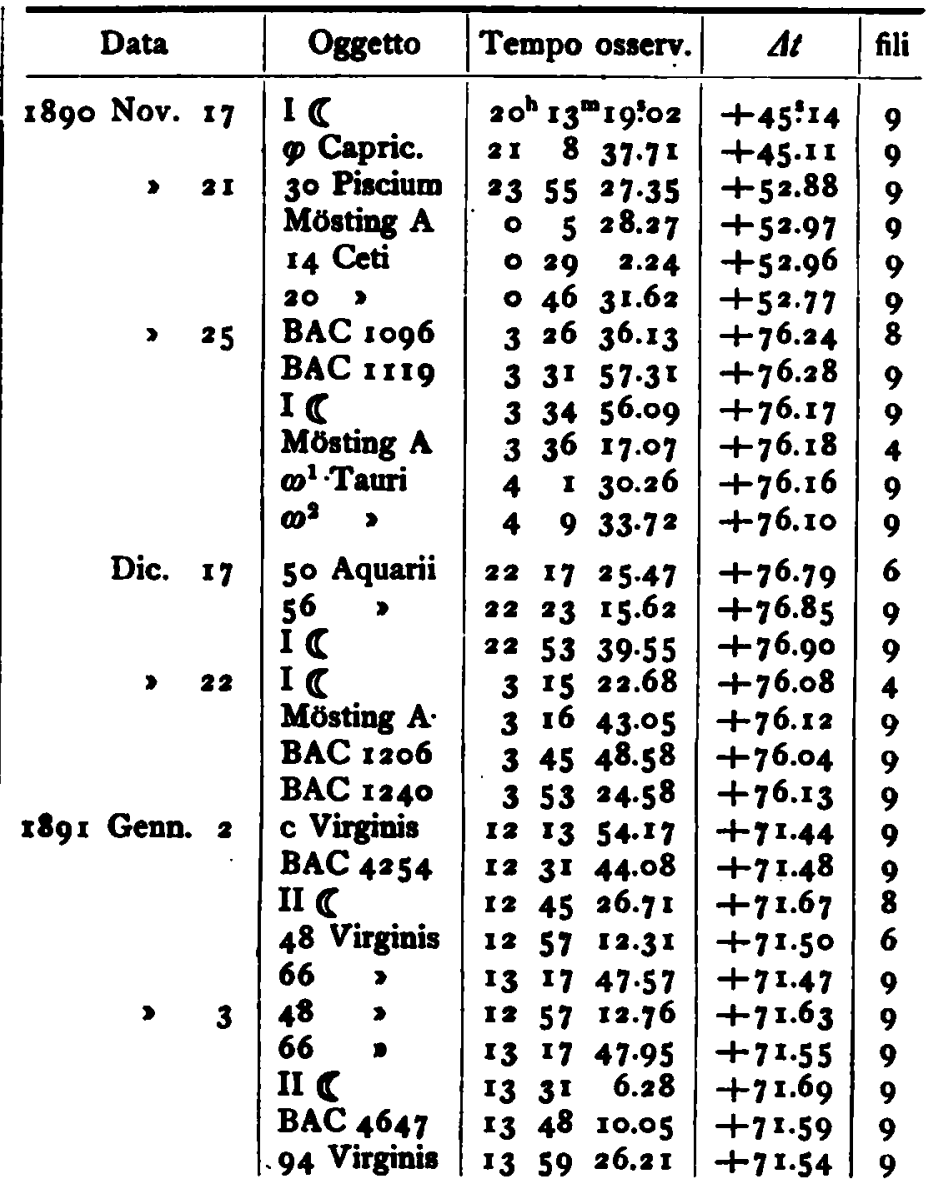


Ascensioni rette del centro della Luna dedotte dal queste osservazioni.

\begin{tabular}{|c|c|c|c|c|c|c|}
\hline Data & t. m. di Tor. & Rid. al Centro & AR. C Centro & $\mathbf{O}-\mathbf{N} . \mathbf{A}$. & Oggetto & Annotazioni \\
\hline $\begin{aligned} 1890 \text { Nov. } & 17 \\
& 21 \\
& 25 \\
& 25\end{aligned}$ & $\begin{array}{rrr}4^{\mathrm{h}} & 28^{\mathrm{m}} & 41 ! 1 \\
8 & 3 & 10.2 \\
11 & 18 & 5.1\end{array}$ & $\begin{array}{rr}+I^{m} & 13: 70^{\circ} \\
-0 & 12.63 \\
+1 & 8.13 \\
-0 & 12.88\end{array}$ & $\begin{array}{rrr}20^{\mathrm{h}} & 15^{\mathrm{m}} & 19: 05 \\
0 & 6 & 9.65 \\
3 & 37 & 22.77 \\
3 & 37 & 22.74\end{array}$ & $\begin{array}{l}-0.07 \\
+0.11 \\
-0.03 \\
-0.06\end{array}$ & $\begin{array}{l}\text { I. Lembo } \\
\text { Mösting A } \\
\text { J. Lembo } \\
\text { Mösting A }\end{array}$ & $\begin{array}{l}\text { luce diurna. } \\
\text { immagine confusa. }\end{array}$ \\
\hline $\begin{array}{rr}\text { Dic. } & 17 \\
: & 22 \\
& 22 \\
1891 \text { Genn. } & 2 \\
& 3\end{array}$ & $\begin{array}{rrr}5 & 10 & 56.6 \\
9 & 12 & 12.8 \\
& & \\
17 & 55 & 16.8 \\
18 & 36 & 51.7\end{array}$ & $\begin{array}{rr}+1 & 9.02 \\
+1 & 7.12 \\
-0 & 13.35 \\
-0 & 1.79 \\
-0 & 1.79\end{array}$ & $\begin{array}{rrr}22 & 55 & 58.25 \\
3 & 17 & 36.89 \\
3 & 17 & 36.89 \\
12 & 45 & 28.87 \\
13 & 31 & 7.17\end{array}$ & $\begin{array}{l}+0.01 \\
+0.03 \\
-0.07 \\
-0.22 \\
-0.39\end{array}$ & & luce diurna, immagine debole. \\
\hline
\end{tabular}

Torino 1891 Genaaio 21 .

\section{T. Aschieri.}

Beobachtungen des Planeten (279) Thule

am Fadenmikrometer des 18 zöll. Refractors der Sternwarte in Strassburg von Dr. H. Kobold.

\begin{tabular}{|c|c|c|c|c|c|c|c|c|c|}
\hline 1891 & M.Z.S & $\Delta a$ & $\Delta \delta$ & Vgl. & $\alpha$ app. & $\log p .4$ & $\delta$ app. & $\log p \cdot 4$ & Red. ad 1. app. \\
\hline $\begin{array}{l}\text { n. } 3 \\
\text { br. } 9 \\
10\end{array}$ & 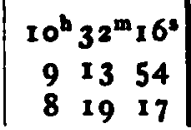 & 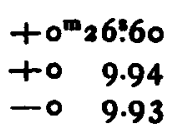 & 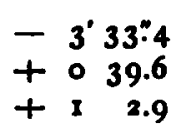 & $\begin{array}{l}18.6 \\
12.12 \\
20.20\end{array}$ & $\begin{array}{lll}6^{\mathrm{h}} & 5^{8 \mathrm{~m}_{2}} & 8.05 \\
6 & 37 & 37.94 \\
6 & 37 & 18.06\end{array}$ & & $\begin{array}{l}+24^{\circ} 11^{\prime} 43^{\prime \prime} 6 \\
+2440 \\
+2440 \\
+240.0\end{array}$ & & $\begin{array}{l}+0: 39+1: 6 \\
+0.44+31 \\
+0.43+3.2\end{array}$ \\
\hline
\end{tabular}

Mittlere Oerter der Vergleichsterne far 189 r.0.

\begin{tabular}{|c|c|c|c|}
\hline$\bullet$ & $\alpha 1891.0$ & $\delta$ I891.0 & Autorität \\
\hline 2 & $\begin{array}{lrr}6^{h} & 5^{8^{m}} & 18.06 \\
7 & 0 & 38.86 \\
6 & 37 & 27.56\end{array}$ & $\begin{array}{r}+24^{\circ} 15^{\circ} 15.4 \\
+242011.8 \\
+243924.3\end{array}$ & $\begin{array}{l}\text { Angeschlossen an } \\
\text { Berlin AG. } \\
\text { Berlin AG. }\end{array}$ \\
\hline
\end{tabular}

\section{H. Kobold.}

Elemente der Planeten (308) und (309).

Planet (308).

Aus 4 Oertern: 3 1. März Marseille, I 3. April Nizza, 27. April und 5. Mai Wien:

Epoche 1891 Mai 5.5 M. Z. Berlin

$$
\begin{aligned}
& M=170^{\circ} 5^{\circ} 24^{\prime \prime} 3 \\
& \omega=2022049.2 \\
& \delta \zeta=182 \text { 21 } 49.0 \\
& i=43 \mathrm{r} \times 3.9 \\
& \varphi=\text { I } 34.25 .1 \\
& \mu=786.547 \\
& \log a=0.436188
\end{aligned}
$$

Uebrig bleibende Fehler:
2) $+0.2+3.2$;
3) $-1.5+1.0$

Planet (309).

Aus 4 Oertern: April 6, 12, 28, Mai I 1 Wien :

Epoche 1891 Mai I r.5 M. Z. Berlin.

$$
\begin{aligned}
& M=239^{\circ} 5^{\prime} 5^{8 \prime \prime} \circ \\
& \omega=\begin{array}{lll}
33^{2} & 8 & 5.8
\end{array} \\
& \Omega=35752 \quad 15.2 \quad \text { M. Aequ. } 1891.0 \\
& i=356 \quad 18.0 \\
& \varphi=5 \text { I } 56.0 \\
& \mu=831.679 \\
& \log a=0.420034
\end{aligned}
$$

$$
\text { 2) }-0.2+1.9 ; \quad 3)-0.3+2.6
$$

Rastatt 189 I Mai I6. A. Berberich.

Berichtigung zu Nr. 3021 Bd. 126 S. 333. In der Formel für $V$ ist $s$ an Stelle von $p$ zu setzen.

Inhalt zu Nr. 3037. C. Fritchard. Parallax of $\beta$ Aurigae. 201. - F. Kleiber. Ueber die mittlere Entfernung derjenigen Sterne, deren eigene Bewegung im Visionsradius bekannt ist. 209. - G. E. Hale. Solar Prominence Photography. 21 1. - T. Aschieri. Osservazioni lunari. 213. - H. Kobold. Beobachtungen des Planeten (279) Thule. 215. - A. Berberich. Elemente der Planeten (308) und (309). 215. - Berichtigung. 215. 